* Corresponding author E-mail address:msenthil_kumar@hotmail.com (M. Senthil Kumar)

Article information Article history: AMS-Volume15-No.3-00121-11 Received 12 April 2011 Accepted 12 May 2011

\section{Experimental Investigations on Cavitation in a Kaplan Turbine}

\author{
Mouleeswaran Senthil Kumar*, S. Chidambara Raja, M. N. Sarath Kumar
}

Department of Mechanical Engineering, PSG College of Technology, Coimbatore-641004, India

\section{BIOGRAPHICAL NOTES}

Mouleeswaran Senthil Kumar, is currently working as Assistant Professor in the Department of Mechanical Engineering at PSG College of Technology, Coimbatore, India. He obtained his B.E. Degree in Mechanical Engineering (1994), M.E. in Engineering Design (1996) and Ph.D. in Active Suspension System (2008). He has about 14 years of teaching experience and 1 year of industrial experience. He has authored 32 journal papers and about 40 conference papers. He received ISTE award for Best Research in the field of Machine design and Vibration by Young Teacher in 2006. He has also received AICTE Career Award for Particle Damping in 2009. He has visited countries such as Portugal, United Kingdom and France for joint research works. He has completed two sponsored projects on Composite Leaf Springs and Propeller Shafts for Automotive Applications and Active Suspension System for Light Passenger Vehicles. Currently he has been the Principal Investigator of two projects in the areas of Vibration Control using Smart Structures. He has been actively involved in many consultancy works in the areas of design and vibration control. His fields of interest include vibration control, composites, smart structures, etc.

Chidambara Raja. S., is currently doing his B.E Mechanical sandwich in PSG College of Technology, Coimbatore, Tamil Nadu, India. The author is a chairman of ASME, students section in PSG College of Technology. He has got about 8 proficiency awards for his excellent academic performance. He has been honoured with Giovanni Volpi award for two times. He has conducted training programme on Design of automation systems for Ashok Leyland Graduate Engineering Trainees. The author has conducted many seminars for students on Pneumatic circuit design, Sensor interfacing using LabVIEW, etc. He has attended indo german workshop on Microsystems for automotive and machine tool industries and national workshop on Issues in Intelligent manufacturing systems and evolving a developmental framework. He has volunteered a national conference on Energy Efficient Mechanical systems Design and Manufacturing. He has presented paper on Cavitation in turbomachinery at SKIT, Jaipur. He had done projects in leading industries like Rane Engine Valves, Chennai on fixing the problem of overall reduction of valves and Ashok Leyland, Hosur on Automation of sheet metal feeding, positioning, ejection and its removal. The author has presented a Conceptual design of Autonomous Under Vehicle (AUV) in the Student Autonomous Competition conducted by National Institute of Ocean Technology, Central Ministry of Earth Sciences. The research interests of the author are Fluid mechanics and Heat and Mass transfer.

Sarath Kumar M.N., is currently doing his B.E. Mechanical Sandwich in PSG College of Technology, Coimbatore, Tamil Nadu, India. The author is the secretary of Association of Mechanical Engineering. He had done projects in leading industries like Rane En- 
gine Valves, Chennai on fixing the problem of overall reduction of valves and Ashok Leyland, Hosur on Automation of sheet metal feeding, positioning, ejection and its removal. He has volunteered a national conference on Energy Efficient Mechanical systems Design and Manufacturing. The author has presented a Conceptual design of Autonomous Under Vehicle (AUV) in the Student Autonomous Competition conducted by National Institute of Ocean Technology, Central Ministry of Earth Sciences. He has conducted training programme on Design of automation systems for Ashok Leyland Graduate Engineering Trainees. The primary field of interest of the author is in the field of Mechatronics and Automation.

\section{KEY WORDS}

Cavitation, Kaplan Turbine, Vibration

\section{ABSTRACT}

An experimental investigation has been carried out in order to evaluate the detection of cavitation in Kaplan turbine. The methodology is based on the analysis of structural vibrations and sound level measured in the turbine. The proposed techniques have been checked in horizontal dispositioned Kaplan turbine suffering from different types of cavitation. Although cavitation within pumps has been the subject of extensive research up to now, as demonstrated by the works from [1], [2], [3], [4] and others, it must be noted that few studies have been published related to cavitation within hydropower turbines. Because of that, the current paper is mainly focused in detection of cavitation in kaplan turbines. First, different causes of vibration in the Kaplan turbine model which has been selected for the experiment are discussed. The work presented here is focused on the most important ones which are the leading edge cavitation due to its erosive power, the bubble cavitation because it affects the machine performance and the draft tube swirl that limits the operation stability and vortex cavitation which is the dominating type of cavitation in Kaplan turbine. Cavitation detection is based on the previous understanding of the cavity dynamics and its location inside the machine. The main techniques are the study of the high frequency spectral content of the signals and of their amplitude demodulation for a given frequency band. Moreover, low frequency spectral content can also be used in certain cases. The results obtained for the various load conditions and speed found in the selected machine are presented and discussed in detail in the paper. Conclusions are drawn about the best sensor, measuring location, signal processing and analysis for each type of cavitation, which serve to validate and to improve the detection techniques.

\section{NOMENCLATURE}

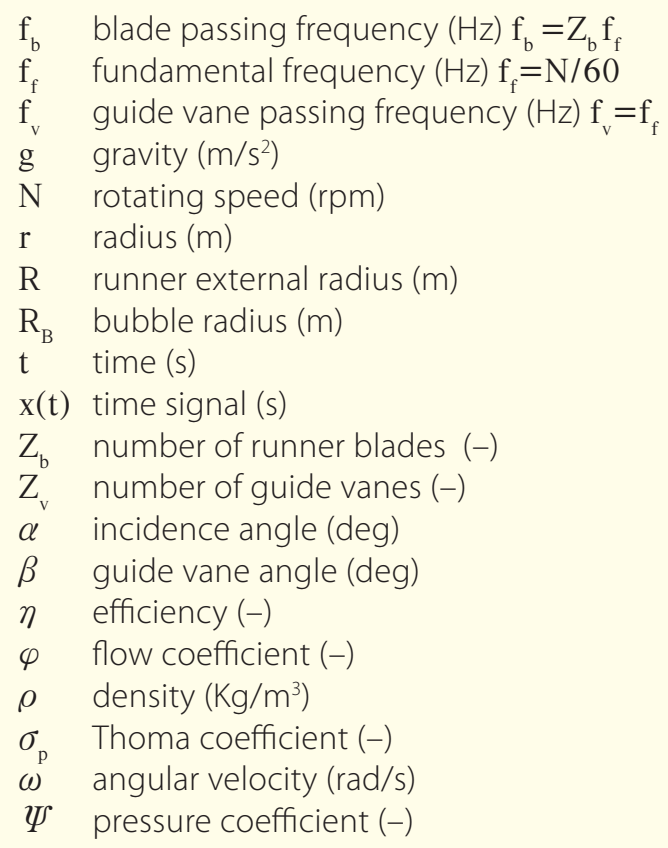

\section{Introduction}

Cavitation is defined as the condition when a liquid reaches a state at which vapour cavities are formed and grow due to dynamic-pressure reductions to the vapour pressure of the liquid at constant temperature. In a flowing liquid, these cavities are subjected to a pressure increase that stops and reverses their growth, collapsing implosively and disappearing. The violent process of cavity collapse takes place in a very short time of about several nanoseconds and results in the emission of large amplitude shock-waves. A high-speed reentrant liquid micro-jet directed towards the boundary can also occur for cavities collapsing close to a solid surface. If the amplitude of the resulting pressure pulse is larger than the limit of the material mechanical strength, a hollow or indentation of several micrometers called "pit" will be formed on the surface. If an accumulation of pits takes place in a narrow area, the material is finally eroded and 
mass loss occurs due to the repetitive action of the cavity collapses. In a flowing liquid, these cavities can take different forms that can be described as travelling bubbles, attached cavities or cavitating vortices.

\section{Types of Cavitation}

The main forms of cavitation that can arise on hydraulic turbines are briefly described in the following paragraphs. More details can be found in $\mathrm{Li}$ [18].

\subsection{Leading Edge Cavitation}

As shown in the Fig1. A. It takes the form of an attached cavity on the suction side of the runner blades due to operation at a higher head than the machine design head $\left(\Psi / \Psi_{\mathrm{A}}>1\right)$ when the incidence angle of the inlet flow is positive and largely deviated from the design value $(\alpha>0)$. It can also occur on the pressure side during operation at a lower head than the machine design head $\left(\Psi / \Psi_{\mathrm{A}}<1\right)$ when the incidence angle is negative $(\alpha<0)$. If unstable, this is a very aggressive type of cavitation that is likely to deeply erode the blades and to provoke pressure fluctuations. Its effect on blade is marked as 1 in Fig. 2.

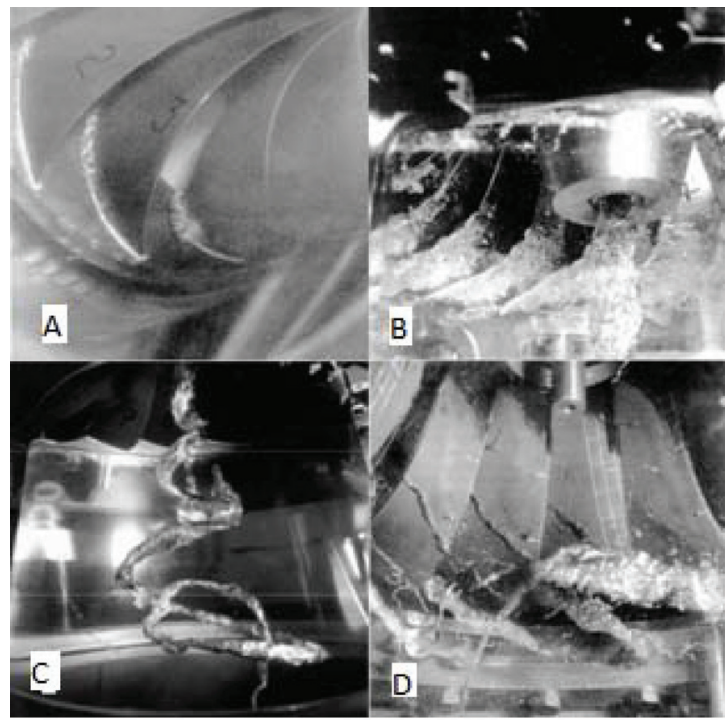

Fig. 1: Various types of Cavitations.

\subsection{Travelling Bubble Cavitation}

As illustrated in Fig. 1. B. It takes the form of separated bubbles attached to the blade suction side near the mid-chord next to the trailing edge. These travelling bubbles appear due to a low plant cavi- tation number sp and they grow with load reaching their maximum when the machine operates in overload condition with the highest flow rate $\left(\varphi / \varphi_{\mathrm{A}}>>1\right)$. This is a severe and noisy type of cavitation that reduces significantly the machine efficiency and that can provoke erosion if the bubbles collapse on the blade. Its effect on blade is indicated as 2 in Fig. 2

\subsection{Draft Tube Swirl}

As demonstrated in Fig. 1. C. It is a cavitation vortex-core flow that is formed just below the runner cone in the centre of the draft tube. Its volume depends on sp and it appears at partial load $(\varphi)$ $\left.\varphi_{\mathrm{A}}<1\right)$ and at overload $\left(\varphi / \varphi_{\mathrm{A}}>1\right)$ due to the residual circumferential velocity component of the flow discharged from the runner. This vortex rotates in the same direction as the runner at part load and in the opposite direction at overload. From 50\% up to $80 \%$ of the best efficiency flow rate, the vortex core takes a helical shape and presents a precession rotation at $0.25-0.35$ times the runner rotating speed. In this case, circumferential pressure pulsations are generated at this low frequency. Strong fluctuations may occur if the precession frequency matches one of the free natural oscillation frequencies of the draft tube or penstock. This provokes large bursts of pressure pulses in the draft tube causing strong vibrations on the turbine and even on the powerhouse. Beyond the best efficiency point the vortex is axially centered in the draft tube cone. Its effect on blade is shown as 3 in Fig. 2.

\subsection{Inter-Blade Vortex Cavitation}

As seen from Fig. 1. D. This is formed by secondary vortices located in the channels between blades that arise due to the flow separation provoked by the incidence variation from the hub to the band. They can attach to the intersection of the blade inlet-edge with the crown or mid-way of the crown between the blades close to the suction side. Only if their tip is in touch with the runner surface they can result in erosion. These vortices appear at partial load operation $\left(\varphi / \varphi_{\mathrm{A}}<1\right)$ and yield a high broadband noise level. They can also appear and cavitate at extremely high-head operation ranges $\left(\Psi / \Psi_{\mathrm{A}}>>1\right)$ because the op is relatively low. In this case, they become unstable and cause strong vibrations. Its effect on blade is marked as 4 in Fig. 2. 


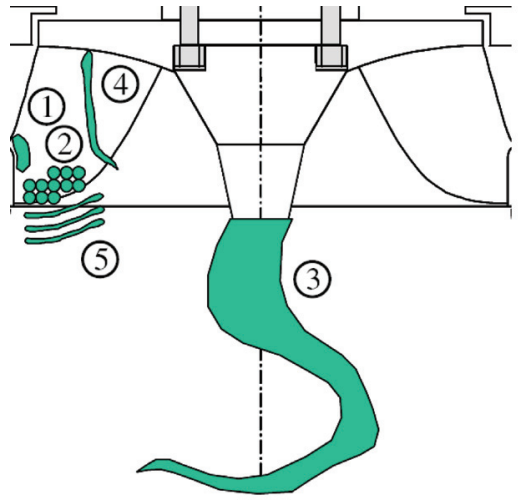

Fig. 2: Effect of different types of cavitation on the blades.

\subsection{Von Karman Vortex Cavitation}

As shown as 5 in Fig2. From the trailing edge of blades and vanes periodic vortex-shedding can occur.

Severe pulsations and singing noise can be caused if a lock-in phenomenon occurs. As a result, the trailing edge might be damaged. For Kaplan turbines, leading edge cavitation is less likely to occur than in Francis turbines. This is because the runner blades have a variable pitch and the machine always operates in "oncam" condition. This means that a is close to the optimum value over a wide operating range thanks to the good combination between runner blade inlet angle and guide vane angle. For the same reason, draft tube swirl is also less severe than in Francis turbines. Travelling bubble cavitation can take place too on the blades suction side due to heavy blade loading. An exclusive type of cavitation of Kaplan turbines is tip vortex cavitation. This cavitation arises in the gap or clearance between the blade tip and the casing. It is a strong type of cavitation that damages an area along the mid-chord length on the periphery of the blade suction side because the vortex tip touches the blade surface. The area on the blade tip end can also suffer erosion.

\section{Experimental Setup}

An experiment on Kaplan turbine has been conducted using the accelerometers and found out the vibrations formed, which is a measure of the cavitations formed in the turbine. The specifications of the turbine and the measuring instruments are shown below. The Fig. 2 shows the experimental setup along with the position of the accelerometers. Fig. 3 shows the sound meter used for the measuring of sound level produced in $\mathrm{dB}$.

\subsection{Turbine setup}

Kaplan Turbine in fluid machinery lab in PSG College of technology, Coimbatore - 04 .

- Discharge: $5000 \mathrm{lpm}$

- Design speed: $1500 \mathrm{rpm}$

- Power: $3.7 \mathrm{~kW}$

- Head: $7 \mathrm{~m}$

\subsection{Measuring Instruments}

The Accelerometer type used is DYTRAN 3097A2, Microphone B\&K 4188 A021.

A DYTRAN model 3097A2 Accelerometer of sensitivity $100 \mathrm{mv} / \mathrm{g}$ is used to measure the amplitude of vibrations in the turbine.

A B\&K model 4188A021 microphone of combined sensitivity $30.5 \mathrm{~dB}$ is used to measure the amplitude of the sound level in the turbine.

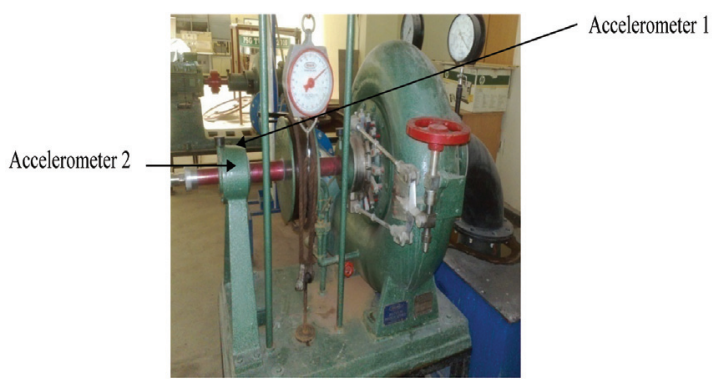

Fig. 3: Experimental Setup.

\subsection{Analysis of the Experiment}

Figure 4 shows how the amplitude varies with the time when the turbine is not subjected to any loads. You can see that the amplitude of the vibrations is large when no load is applied to the turbine because of the huge water fluctuations along the rotor blades. From the Figs. 4 and 5, it is shown that the vibrations are reduced in half discharge conditions as there will be less water in the casing.

Figures 5, 6 \& 7 shows how the vibrations are getting reduced by the addition of the load proving that the frequency of vibrations is inversely proportional to the square root of the mass. Fig. 8 shows the LabVIEW program containing the block diagram for the experiment.

It can be seen that the sound also gets reduced as a consequence of the reduction in the amplitude of vibrations from the Fig. 9. And the vibrations are at peak in the free running conditions and gradually decrease as the loads are increased as shown in the Fig. 10. 


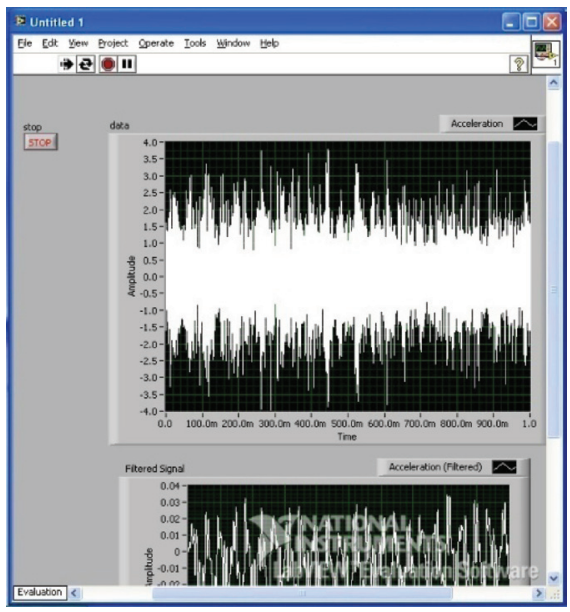

Fig. 4: Amplitude of vibrations ( $\mathrm{mm}$ ) for No Load under full discharge conditions (Radial Position - 1).

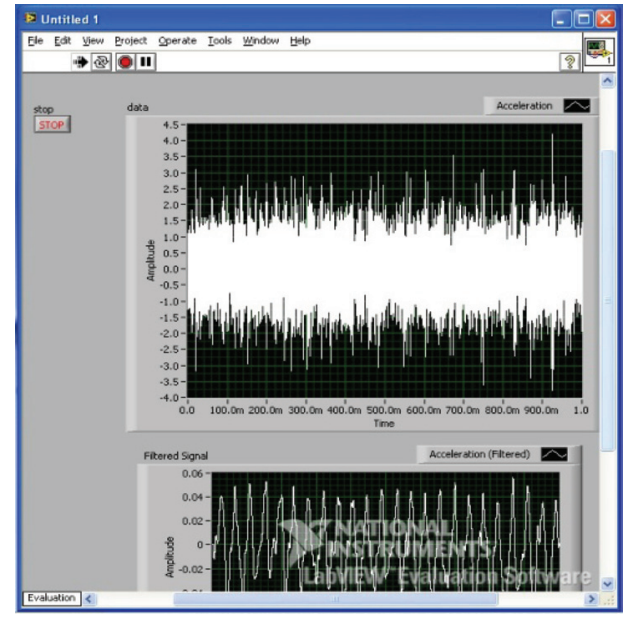

Fig. 5: Amplitude of vibrations $(\mathrm{mm})$ for no Load under Half Discharge conditions (Radial Position - 1).

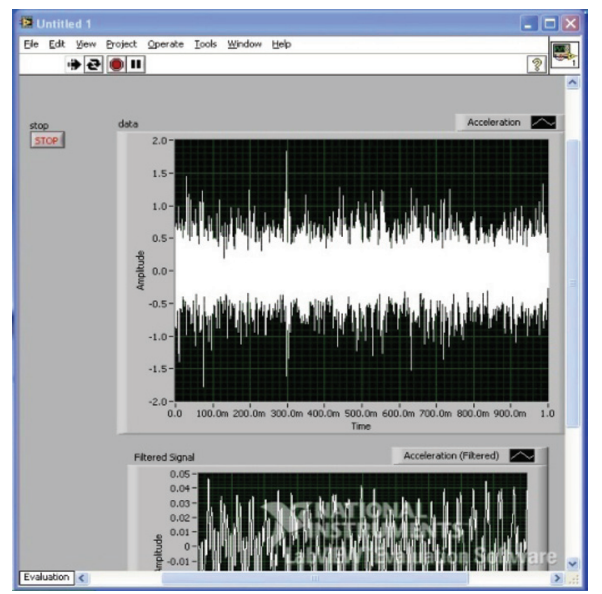

Fig. 6: Amplitude of vibrations ( $\mathrm{mm}$ ) for $5 \mathrm{~kg}$ load (Radial Position $-2)$.

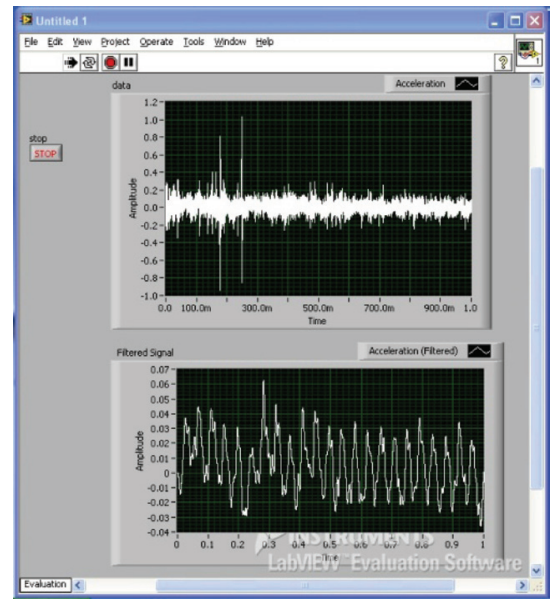

Fig. 7: Amplitude of vibrations ( $\mathrm{mm}$ ) for $10 \mathrm{~kg}$ Load (Radial Position - 2).

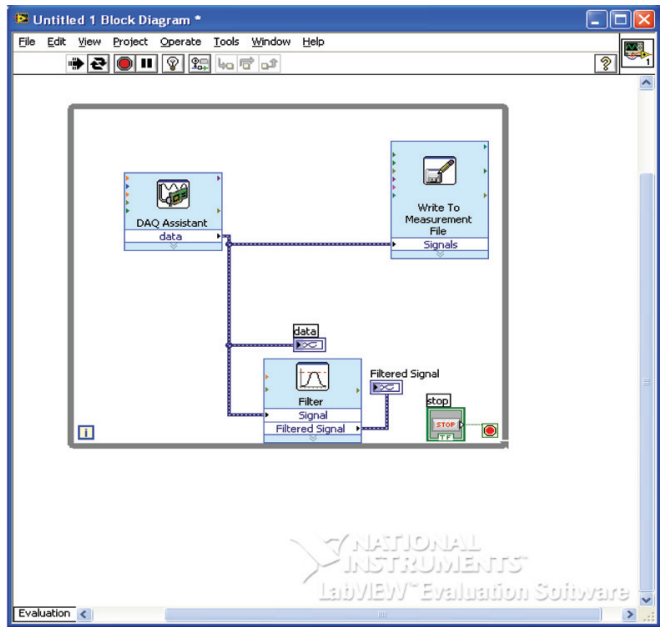

Fig. 8: Amplitude of vibrations ( $\mathrm{mm}$ ) for $15 \mathrm{~kg}$ Load (Radial Position - 2).

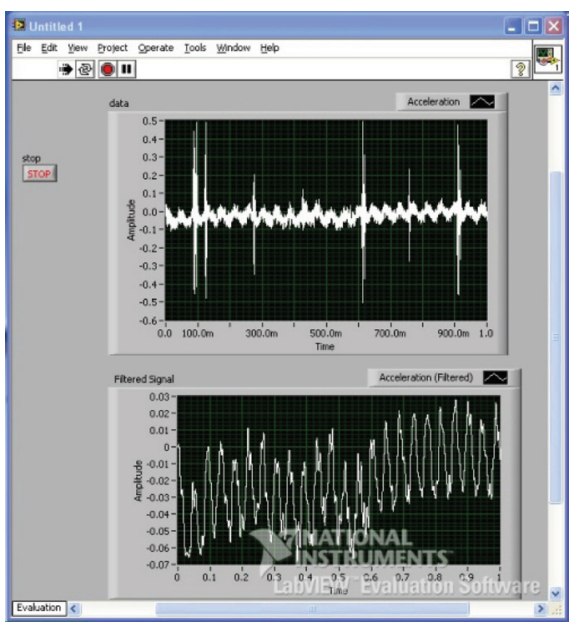

Fig. 9: LabVIEW program for our Experiment. 


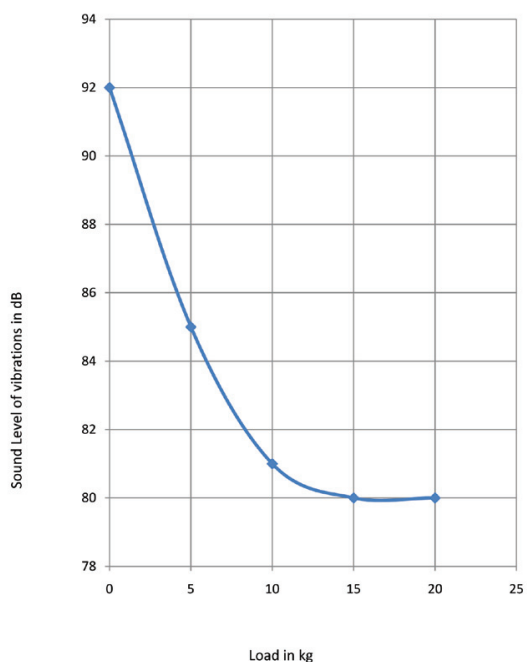

Fig. 10: Sound level of the vibrations $(d B)$ for the various loads

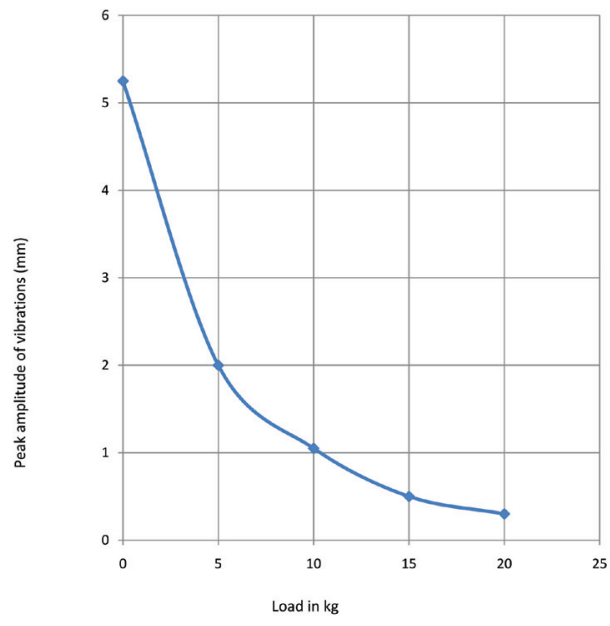

Fig. 11: Peak amplitude of vibrations $(\mathrm{mm})$ for various loads.

\subsection{Blade Erosion}

The blade erosion due the effects of cavitation is shown in the Fig. 11.

\subsection{Calculation of Frequency}

A fundamental frequency $\left(\mathrm{f}_{\mathrm{f}}\right)$ of $38.33 \mathrm{~Hz}$ is estimated from the experiment at worst case condition (high speed condition). A guide vane passing frequency $\left(\mathrm{f}_{\mathrm{v}}=\mathrm{Z}_{\mathrm{v}} \mathrm{f}_{\mathrm{f}}\right)$ is calculated as $306.33 \mathrm{~Hz}$. A runner blade passing frequency $\left(\mathrm{f}_{\mathrm{b}}=\mathrm{Z}_{\mathrm{b}} \mathrm{f}_{\mathrm{f}}\right)$ is found to be $153.33 \mathrm{~Hz}$.

\section{Conclusion}

The amplitude of these frequency peaks is related to the intensity of the cavitation. When load increases, amplitude of vibration decreases as frequency of

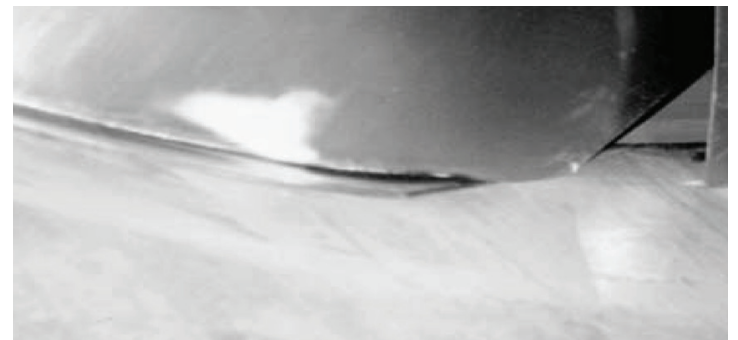

Fig. 12: Sound level of the vibrations (dB) for the various loads.

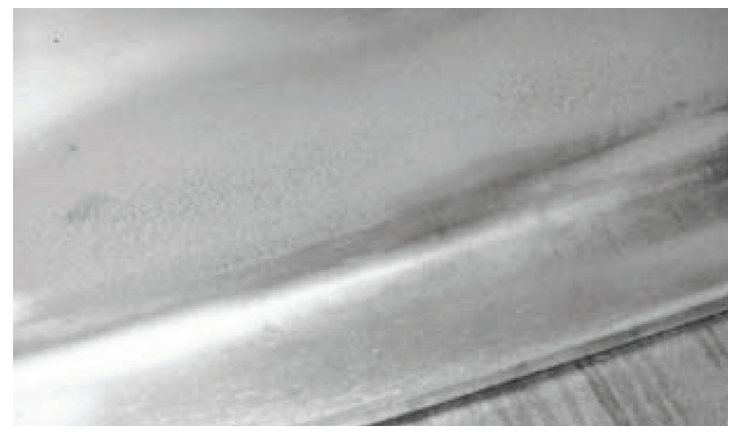

Fig. 13: Peak amplitude of vibrations $(\mathrm{mm})$ for various loads.

vibration is inversely proportional to mass. When speed increases above the design speed, amplitude increases and it suppresses the performance of the turbine.

The suggested methods to improve the performance of the kaplan turbine are change material of the turbine, operate turbine at the design discharge and speed, avoid operating turbine at peak frequencies, ensure complete balancing of turbine (both static and dynamic) thereby avoiding vibration at source.

\section{References}

[1] Coutier-Delgosha, O., Fortes-Patella, R., Reboud, J.L., Hofmann, M., Stoffel, B. (2003). Experimental and numerical studies in a centrifugal pump with two-dimensional curved blades in cavitating condition, Journal of Fluids Engineering, vol. 125, pp. 970-978

[2] Cudina, M. (2003). Detection of cavitation phenomenon in a centrifugal pump using audible sound, Mechanical Systems and Signal Processing, vol. 17, no. 6, pp. 1335-1347

[3] Baldassarre, A., De Lucia, M., Nesi, P. (1998). Real-time detection of cavitation for hydraulic turbomachines, Real-Time Imaging, vol. 4, pp. 403-416

[4] McNulty, P.J., Pearsall, I.S. (1982). Cavitation inception in pumps, Journal of Fluids Engineering, vol. 104, pp. 99-104

[5] Avellan, F., Henry, P. (1987). Towards the prediction of cavitation erosion: IMHEF research program, Proceedings of the 
EPRY Symposium on Power Plant Pump, New Orleans, pp. 1-22

[6] Bourdon, P., Simoneau, R., Lavigne, P. (1989). A vibratory approach to the detection of erosive cavitation, Proceedings of the Third International Symposium on Cavitation Noise and Erosion in Fluid Systems, FED-vol. 88, ASME Winter Annual Meeting, San Francisco, CA, pp. 103-109

[7] Knapp, R.T., Daily, J.W., Hammit, F.G., Cavitation, McGraw-Hill, New York, 1970

[8] Avellan, F., Farhat, M. (1989). Shock pressure generated by cavitation vortex collapse, Proceedings of the Third International Symposium on Cavitation Noise and Erosion in Fluid Systems, FED-vol. 88, ASME Winter Annual Meeting, San Francisco, CA, pp. 119-125

[9] Philipp, A., Lauterborn, W. (1998). Cavitation erosion by single laser-produced bubbles, Journal of Fluid Mechanics, vol. 361, pp. 75-116

[10] Hammit, F.G. (1979). Cavitation erosion: the state of the art and predicting capability, Applied Mechanics Reviews, vol. 32, no. 6, pp. 665-675

[11] Li (Ed.), S.C. (2000). Cavitation of Hydraulic Machinery, vol. 1, Imperial College Press, London

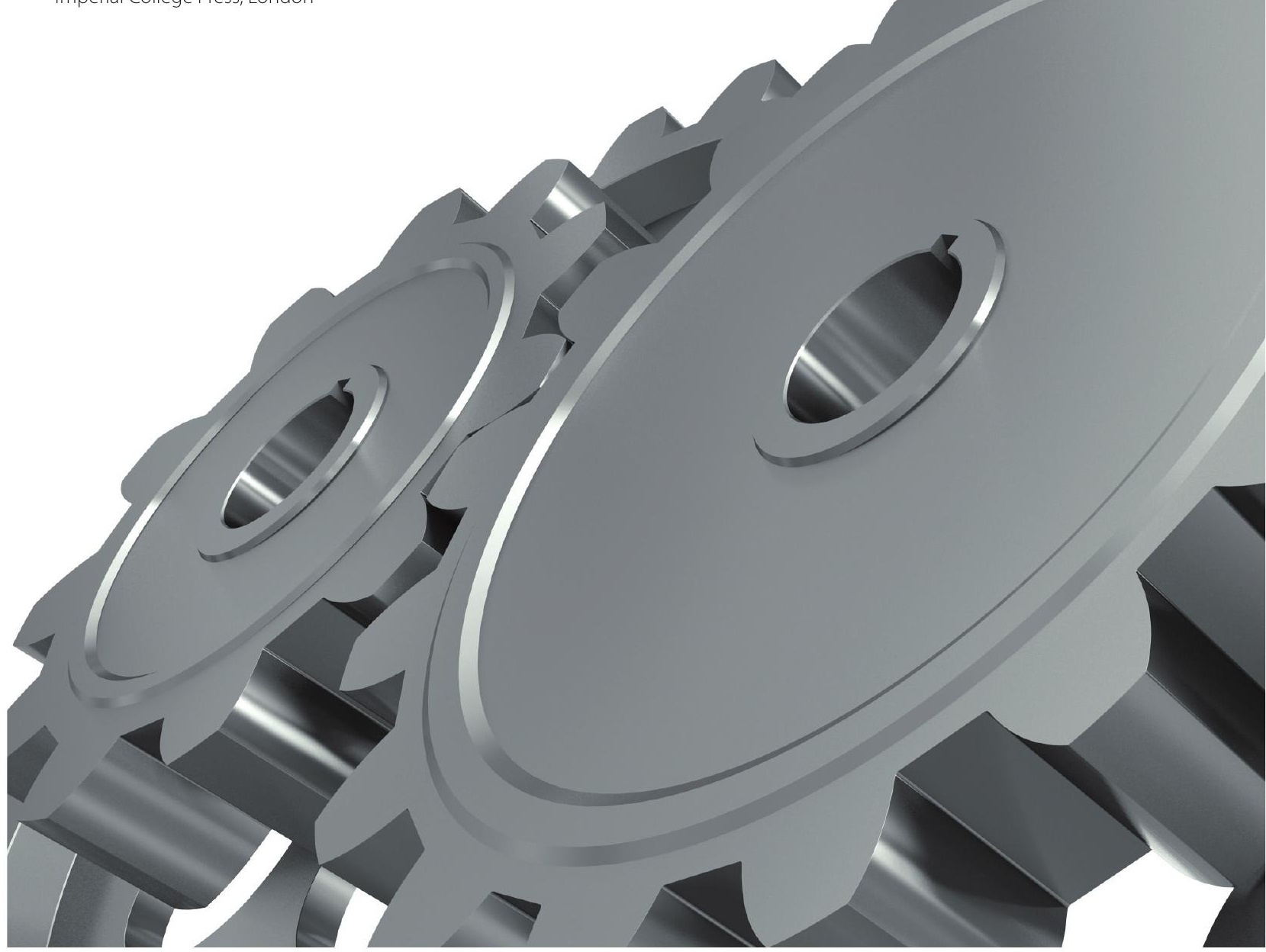

